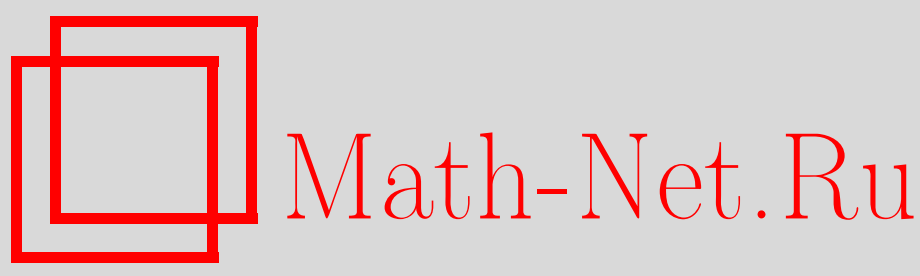

M. Х. Бештоков, Краевые задачи для нагруженных псевдопараболических уравнений дробного порядка и разностные методы их решения, Изв. вузов. Матем., 2019, номер 2, 3-12

DOI: https://doi.org/10.26907/0021-3446-2019-2-3-12

Использование Общероссийского математического портала Math-Net.Ru подразумевает, что вы прочитали и согласны с пользовательским соглашением

http://www.mathnet.ru/rus/agreement

Параметры загрузки:

IP: 54.162 .127 .20

26 апреля 2023 г., $07: 45: 16$

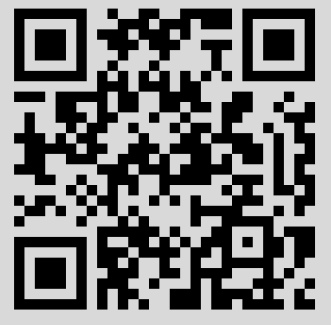


Известия вузов. Математика 2019, № 2, c. 3-12 https://kpfu.ru/science/nauchnye-izdaniya/ivrm/ e-mail: izvuz.matem@kpfu.ru

\title{
М.Х. БЕШТОКОВ
}

\section{КРАЕВЫЕ ЗАДАЧИ ДЛЯ НАГРУЖЕННЫХ ПСЕВДОПАРАБОЛИЧЕСКИХ УРАВНЕНИЙ ДРОБНОГО ПОРЯДКА И РАЗНОСТНЫЕ МЕТОДЫ ИХ РЕШЕНИЯ}

\begin{abstract}
Аннотация. Работа посвящена локальным и нелокальным краевым задачам для нагруженного дифференциального уравнения с переменными коэффициентами и с дробной производной Герасимова-Капуто. Для решения рассматриваемых задач получены априорные оценки в дифференциальной и разностной трактовках, из чего следуют единственность и устойчивость решения по начальным данным и правой части, а также сходимость решения разностной задачи к решению дифференциальной задачи.
\end{abstract}

Ключевые слова: краевые задачи, априорная оценка, нагруженные уравнения, уравнение псевдопараболического типа, дифференциальное уравнение дробного порядка, дробная производная Герасимова-Капуто.

УДК: 519.63

DOI: $10.26907 / 0021-3446-2019-2-3-12$

Введение. Важным разделом в теории дифференциальных уравнений являются нагруженные уравнения. Первые работы были посвящены нагруженным интегральным уравнениям. Этому классу нагруженных уравнений посвящены работы А. Кнезера, Л. Лихтенштейна, Н.Н. Назарова, А.Ш. Габиб-Заде. Важность изучения таких уравнений подчеркивали А.Н. Крылов, В.И. Смирнов, А.Н. Тихонов, А.А. Самарский, которые приводили примеры прикладных задач из техники и физики, сводящиеся к нагруженным интегральным уравнениям.

Большой вклад в разработку теории нагруженных дифференциальных уравнений внесли: В.М. Будак, А.Д. Искендеров, А.М. Нахушев, В.М. Казиев, А.М. Крал. В обзорных работах A.M. Нахушева на многочисленных примерах показана практическая и теоретическая важность исследований нагруженных дифференциальных уравнений. Одним из методов приближенного решения краевых задач для дифференциальных уравнений является предложенный А.М. Нахушевым метод редукции интегро-дифференциальных уравнений к нагруженным дифференциальным уравнениям. В работе [1] впервые указана связь нелокальных задач с нагруженными уравнениями. Нелокальные задачи типа Бицадзе-Самарского для уравнений Лапласа и теплопроводности эквивалентно редуцированы к локальным задачам для нагруженных дифференциальных уравнений.

Краевые задачи для нагруженных псевдопараболических уравнений рассматривались в [2]. Задачи расчета тепломассообмена с сосредоточенными источниками (стоками) переносимой субстанции [3] и, подобно [4], [5], задачи регулирования уровня грунтовых вод при орошении приводят к необходимости исследования краевых задач для нагруженных псевдопараболических уравнений.

Поступила в редакцию 17.01.2018, после доработки 14.06.2018. Принята к публикации 20.06.2018 
Разностным методам решения краевых задач для вырождающихся псевдопараболических уравнений с дробной производной Герасимова-Капуто посвящена работа автора [6]. Данная статья является непосредственным продолжением [6].

1. Постановка задачи. В замкнутом цилиндре $\bar{Q}_{T}=\{(x, t): 0 \leq x \leq l, 0 \leq t \leq T\}$ рассмотрим первую краевую задачу для нагруженного псевдопараболического уравнения с дробной производной Герасимова-Капуто порядка $\alpha$

$$
\begin{gathered}
\partial_{0 t}^{\alpha} u=\frac{\partial}{\partial x}\left(k(x, t) \frac{\partial u}{\partial x}\right)+\partial_{0 t}^{\alpha} \frac{\partial}{\partial x}\left(\eta(x) \frac{\partial u}{\partial x}\right)+r(x, t) \frac{\partial u}{\partial x}\left(x_{0}, t\right)-q(x, t) u+f(x, t), \\
0<x<l, \quad 0<t \leq T, \\
u(0, t)=u(l, t)=0, \quad 0 \leq t \leq T, \\
u(x, 0)=u_{0}(x), \quad 0 \leq x \leq l,
\end{gathered}
$$

где

$$
0<c_{0} \leq k(x, t), \eta(x) \leq c_{1}, \quad\left|k_{x}(x, t)\right|,\left|\eta_{x}(x)\right|,|r(x, t)|,|q(x, t)| \leq c_{2},
$$

$\partial_{0 t}^{\alpha} u=\frac{1}{\Gamma(1-\alpha)} \int_{0}^{t} \frac{u_{\tau}(x, \tau)}{(t-\tau)^{\alpha}} d \tau-$ дробная производная в смысле Герасимова-Капуто порядка $\alpha$ [7], $0<\alpha<1, x_{0}$ - произвольная точка интервала $[0, l], \partial_{0 t}^{\alpha} u=D_{0 t}^{\alpha} u-\frac{u(0)}{\Gamma(1-\alpha) t^{\alpha}}, D_{0 t}^{\alpha} u=$ $\frac{1}{\Gamma(1-\alpha)} \frac{d}{d t} \int_{0}^{t} \frac{u d \tau}{(t-\tau)^{\alpha}}-$ дробная производная в смысле Римана-Лиувилля порядка $\alpha$.

В дальнейшем будем предполагать, что задача (1.1)-(1.3) имеет единственное решение, обладающее нужными производными. Будем также считать, что коэффициенты уравнения и граничных условий удовлетворяют необходимым условиям гладкости, обеспечивающим нужный порядок аппроксимации разностной схемы.

По ходу изложения будем также использовать положительные постоянные числа $M_{i}$, $i=1,2, \ldots$, зависящие только от входных данных рассматриваемой задачи.

2. Априорная оценка в дифференциальной форме. Для получения априорной оценки решения задачи (1.1)-(1.3) в дифференциальной форме умножим уравнение (1.1) скалярно на $U=\partial_{0 t}^{\alpha} u-u_{x x}$

$$
\left(\partial_{0 t}^{\alpha} u, U\right)=\left(\left(k u_{x}\right)_{x}, U\right)+\left(\partial_{0 t}^{\alpha}\left(\eta u_{x}\right)_{x}, U\right)+\left(r u_{x}\left(x_{0}, t\right), U\right)-(q u, U)+(f, U) .
$$

В дальнейшем изложении скалярное произведение и норма будут определяться как в работе [6].

Из (2.1) после несложных преобразований, пользуясь (1.2), неравенством Коши с $\varepsilon$ ([8], с. 100) и леммой 1 из [9], находим [6]

$$
\begin{aligned}
\left\|\partial_{0 t}^{\alpha} u\right\|_{0}^{2}+ & \left(\frac{1}{2}+\frac{c_{0}}{2}\right) \partial_{0 t}^{\alpha}\left\|u_{x}\right\|_{0}^{2}+\frac{c_{0}}{2} \partial_{0 t}^{\alpha}\left\|u_{x x}\right\|_{0}^{2}+\frac{c_{0}}{2}\left\|\partial_{0 t}^{\alpha} u_{x}\right\|_{0}^{2}+c_{0}\left\|u_{x x}\right\|_{0}^{2} \leq \\
& \leq \varepsilon M_{1}\left\|\partial_{0 t}^{\alpha} u\right\|_{0}^{2}+M_{2}^{\varepsilon}\left(\left\|u_{x}\right\|_{0}^{2}+\left\|u_{x x}\right\|_{0}^{2}\right)+M_{3}^{\varepsilon}\|f\|_{0}^{2} .
\end{aligned}
$$

Выбирая $\varepsilon=\frac{1}{2 M_{1}}$, из $(2.2)$ находим

$$
\partial_{0 t}^{\alpha}\|u\|_{1}^{2}+\left\|u_{x x}\right\|_{0}^{2}+\left\|\partial_{0 t}^{\alpha} u\right\|_{0}^{2}+\left\|\partial_{0 t}^{\alpha} u_{x}\right\|_{0}^{2} \leq M_{4}\|u\|_{1}^{2}+M_{5}\|f\|_{0}^{2},
$$

где $\|u\|_{1}^{2}=\left\|u_{x}\right\|_{0}^{2}+\left\|u_{x x}\right\|_{0}^{2}$.

Применяя к обеим частям неравенства (2.3) оператор дробного интегрирования $D_{0 t}^{-\alpha}$, получаем

$$
\|u\|_{1}^{2}+D_{0 t}^{-\alpha}\left(\left\|u_{x x}\right\|_{0}^{2}+\left\|\partial_{0 t}^{\alpha} u\right\|_{0}^{2}+\left\|\partial_{0 t}^{\alpha} u_{x}\right\|_{0}^{2}\right) \leq M_{6} D_{0 t}^{-\alpha}\|u\|_{1}^{2}+M_{7}\left(D_{0 t}^{-\alpha}\|f\|_{0}^{2}+\left\|u_{0}(x)\right\|_{1}^{2}\right) .
$$


На основании леммы 2 [8] из (2.4) находим искомую априорную оценку

$$
\|u\|_{2}^{2} \leq M_{8}\left(D_{0 t}^{-\alpha}\|f\|_{0}^{2}+\left\|u_{0}(x)\right\|_{1}^{2}\right),
$$

где $\|u\|_{2}^{2}=\|u\|_{1}^{2}+D_{0 t}^{-\alpha}\left(\left\|u_{x x}\right\|_{0}^{2}+\left\|\partial_{0 t}^{\alpha} u\right\|_{0}^{2}+\left\|\partial_{0 t}^{\alpha} u_{x}\right\|_{0}^{2}\right), M_{8}$ - положительная постоянная, зависящая только от входных данных (1.1)-(1.3), $D_{0 t}^{-\alpha} u=\frac{1}{\Gamma(\alpha)} \int_{0}^{t} \frac{u d \tau}{(t-\tau)^{1-\alpha}}-$ дробный интеграл Римана-Лиувилля порядка $\alpha, 0<\alpha<1$.

Из полученной априорной оценки (2.5) следуют единственность и устойчивость решения по начальным данным и правой части в смысле нормы $\|u\|_{2}^{2}$.

Теорема 1. Eсли $k(x, t) \in C^{1,0}\left(Q_{T}\right), \eta(x) \in C^{1}[0, l], r(x, t), q(x, t), f(x, t) \in C\left(Q_{T}\right), u(x, t) \in$ $C^{(2,0)}\left(Q_{T}\right) \cap C^{(1,0)}\left(\bar{Q}_{T}\right), \partial_{0 t}^{\alpha} u(x, t) \in C\left(Q_{T}\right)$ и выполнены условия (1.4), то для решения задачи (1.1)-(1.3) справедлива априорная оценка (2.5).

3. Устойчивость и сходимость разностной схемы. Для решения задачи (1.1)-(1.3) применим метод конечных разностей. В замкнутом цилиндре $\bar{Q}_{T}$ введем равномерную сетку $\bar{\omega}_{h \tau}=\bar{\omega}_{h} \times \bar{\omega}_{\tau}$, где $\bar{\omega}_{h}=\left\{x_{i}=i h, i=\overline{0, N}, h=l / N\right\}, \bar{\omega}_{\tau}=\left\{t_{j}=j \tau, j=0,1, \ldots, j_{0}, \tau=T / j_{0}\right\}$. На равномерной сетке $\bar{\omega}_{h \tau}$ дифференциальной задаче (1.1)-(1.3) поставим в соответствие разностную схему порядка аппроксимации $O\left(h^{2}+\tau^{2}\right)$ :

$$
\begin{gathered}
\Delta_{0 t_{j+\sigma}}^{\alpha} y_{i}=\left(a^{j} y_{\bar{x}}^{(\sigma)}\right)_{x, i}+\Delta_{0 t_{j+\sigma}}^{\alpha}\left(\gamma y_{\bar{x}}\right)_{x, i}+r_{i}^{j}\left(y_{\dot{x}, i_{0}}^{(\sigma)} x_{i_{0}}^{-}+y_{x_{x}, i_{0}+1}^{(\sigma)} x_{i_{0}}^{+}\right)-d_{i}^{j} y_{i}^{(\sigma)}+\varphi_{i}^{j}, \\
y_{0}^{(\sigma)}=y_{N}^{(\sigma)}=0 \\
y(x, 0)=u_{0}(x),
\end{gathered}
$$

где $\Delta_{0 t_{j+\sigma}}^{\alpha} y=\frac{\tau^{1-\alpha}}{\Gamma(2-\alpha)} \sum_{s=0}^{j} c_{j-s}^{(\alpha, \sigma)} y_{t}^{s}-$ дискретный аналог дробной производной ГерасимоваКапуто порядка $\alpha, 0<\alpha<1$, с порядком аппроксимации $O\left(h^{2}+\tau^{3-\alpha}\right)$ [10].

Имеем

$$
\begin{gathered}
a_{0}^{(\alpha, \sigma)}=\sigma^{1-\alpha}, \quad a_{l}^{(\alpha, \sigma)}=(l+\sigma)^{1-\alpha}-(l-1+\sigma)^{1-\alpha}, \quad l \geq 1, \\
b_{l}^{(\alpha, \sigma)}=\frac{1}{2-\alpha}\left[(l+\sigma)^{2-\alpha}-(l-1+\sigma)^{2-\alpha}\right]-\frac{1}{2}\left[(l+\sigma)^{1-\alpha}+(l-1+\sigma)^{1-\alpha}\right], \quad l \geq 1,
\end{gathered}
$$

при $j=0 c_{0}^{(\alpha, \sigma)}=a_{0}^{(\alpha, \sigma)} ;$ при $j>0 c_{s}^{(\alpha, \sigma)}= \begin{cases}a_{0}^{(\alpha, \sigma)}+b_{1}^{(\alpha, \sigma)}, & s=0 ; \\ a_{s}^{(\alpha, \sigma)}+b_{s+1}^{(\alpha, \sigma)}-b_{s}^{(\alpha, \sigma)}, & 1 \leq s \leq j-1 ; \\ a_{j}^{(\alpha, \sigma)}-b_{j}^{(\alpha, \sigma)}, & s=j,\end{cases}$

$$
\begin{gathered}
a_{i}^{j}=k\left(x_{i-0.5}, t^{j+\sigma}\right), \gamma_{i}=\eta\left(x_{i-0.5}\right), \quad \varphi_{i}^{j}=f\left(x_{i}, t^{j+\sigma}\right), \quad \sigma=1-\frac{\alpha}{2}, \quad c_{s-1}^{(\alpha, \sigma)}>c_{s}^{(\alpha, \sigma)}, \\
c_{s}^{(\alpha, \sigma)}>\frac{1-\alpha}{2}(s+\sigma)^{-\alpha}>0 ; \quad y^{(\sigma)}=\sigma y^{j+1}+(1-\sigma) y^{j} ; \quad d_{i}^{j}=d\left(x_{i}, t^{j+\sigma}\right), x_{i_{0}} \leq x_{0} \leq x_{i_{0}+1}, \\
x_{i_{0}}^{-}=\frac{x_{i_{0}+1}-x_{0}}{h}, \quad x_{i_{0}}^{+}=\frac{x_{0}-x_{i_{0}}}{h} .
\end{gathered}
$$

Априорную оценку найдем методом энергетических неравенств, для этого умножим (3.1) скалярно на $\bar{y}=\Delta_{0 t_{j+\sigma}}^{\alpha} y-y_{\bar{x} x}^{(\sigma)}$ :

$$
\begin{aligned}
\left(\Delta_{0 t_{j+\sigma}}^{\alpha} y, \bar{y}\right)=\left(\left(a y_{\bar{x}}^{(\sigma)}\right)_{x}, \bar{y}\right)+ & \left(\Delta_{0 t_{j+\sigma}}^{\alpha}\left(\gamma_{i} y_{\bar{x}}\right)_{x}, \bar{y}\right)+\left(r_{i}^{j}\left(y_{\dot{x}, i_{0}}^{(\sigma)} x_{i_{0}}^{-}+y_{\dot{x}, i_{0}+1}^{(\sigma)} x_{i_{0}}^{+}\right), \bar{y}\right)- \\
& -\left(d y^{(\sigma)}, \bar{y}\right)+(\varphi, \bar{y}) .
\end{aligned}
$$


Из (3.4) с учетом (3.2) и леммы 1 [10] находим [6]

$$
\begin{gathered}
\left.\left.\left\|\Delta_{0 t_{j+\sigma}}^{\alpha} y\right\|_{0}^{2}+\left(\frac{1}{2}+\frac{c_{0}}{2}\right) \Delta_{0 t_{j+\sigma}}^{\alpha} \| y \bar{x}\right]\left.\right|_{0} ^{2}+c_{0}\left\|y_{\bar{x} x}^{(\sigma)}\right\|_{0}^{2}+\frac{c_{0}}{2} \| \Delta_{0 t_{j+\sigma}}^{\alpha} y \bar{x}\right]\left.\right|_{0} ^{2}+\frac{c_{0}}{2} \Delta_{0 t_{j+\sigma}}^{\alpha}\left\|y_{\bar{x} x}\right\|_{0}^{2} \leq \\
\left.\leq \varepsilon M_{1}\left\|\Delta_{0 t_{j+\sigma}}^{\alpha} y\right\|_{0}^{2}+\left.M_{2}^{\varepsilon}\left(\| y_{\bar{x}}^{(\sigma)}\right]\right|_{0} ^{2}+\left\|y_{\bar{x} x}^{(\sigma)}\right\|_{0}^{2}\right)+M_{3}^{\varepsilon}\|\varphi\|_{0}^{2} .
\end{gathered}
$$

Выбирая $\varepsilon=\frac{1}{2 M_{1}}$, из (3.5) находим

$$
\left.\Delta_{0 t_{j+\sigma}}^{\alpha}\|y\|_{1}^{2}+\left\|\Delta_{0 t_{j+\sigma}}^{\alpha} y\right\|_{0}^{2}+\| \Delta_{0 t_{j+\sigma}}^{\alpha} y \bar{x}\right]\left.\right|_{0} ^{2}+\left\|y_{\bar{x} x}\right\|_{0}^{2} \leq M_{4}\left\|y^{(\sigma)}\right\|_{1}^{2}+M_{5}\|\varphi\|_{0}^{2},
$$

где $\left.\| y_{\bar{x}}\right]\left.\right|_{0} ^{2}+\left\|y_{\bar{x} x}\right\|_{0}^{2}=\|y\|_{1}^{2}$.

Перепишем (3.6) в другой форме

$$
\Delta_{0 t_{j+\sigma}}^{\alpha}\|y\|_{1}^{2} \leq M_{6}^{\sigma}\left\|y^{j+1}\right\|_{1}^{2}+M_{7}^{\sigma}\left\|y^{j}\right\|_{1}^{2}+M_{5}\|\varphi\|_{0}^{2} .
$$

На основании леммы 7 [6] из (3.7) получаем

$$
\left\|y^{j+1}\right\|_{1}^{2} \leq M_{8}\left(\left\|y^{0}\right\|_{1}^{2}+\frac{t_{j}^{\alpha}}{\Gamma(1+\alpha)} \max _{0 \leq j^{\prime} \leq j}\left\|\varphi^{j^{\prime}}\right\|_{0}^{2}\right)
$$

где $M_{8}$ - положительная постоянная, не зависящая от $h$ и $\tau$.

Из априорной оценки (3.8) следуют единственность и устойчивость решения задачи (3.1)(3.3) по начальным данным и правой части в смысле нормы $\left\|y^{j+1}\right\|_{1}^{2}$.

Теорема 2. Пусть выполнены условия (1.4), тогда существует такое малое $\tau_{0}=\tau_{0}\left(c_{0}, c_{1}\right.$, $\left.c_{2}, \alpha, \sigma\right)$, что если $\tau \leq \tau_{0}$, то для решения разностной задачи (3.1)-(3.3) справедлива априорная оценка (3.8).

Пусть $u(x, t)$ - решение задачи $(1.1)-(1.3), y\left(x_{i}, t_{j}\right)=y_{i}^{j}-$ решение разностной задачи (3.1)-(3.3). Для оценки точности разностной схемы (3.1)-(3.3) рассмотрим разность $z_{i}^{j}=$ $y_{i}^{j}-u_{i}^{j}$, где $u_{i}^{j}=u\left(x_{i}, t_{j}\right)$. Тогда, подставляя $y=z+u$ в соотношения (3.1)-(3.3), получаем задачу для функции $z$ :

$$
\begin{gathered}
\Delta_{0 t_{j+\sigma}}^{\alpha} z_{i}=\left(a^{j} z_{\bar{x}}^{(\sigma)}\right)_{x, i}+\Delta_{0 t_{j+\sigma}}^{\alpha}\left(\gamma z_{\bar{x}}\right)_{x, i}+r_{i}^{j}\left(z_{\dot{x}, i_{0}}^{(\sigma)} x_{i_{0}}^{-}+z_{\dot{x}, i_{0}+1}^{(\sigma)} x_{i_{0}}^{+}\right)-d_{i}^{j} z_{i}^{(\sigma)}+\Psi_{i}^{j}, \\
z_{0}^{(\sigma)}=z_{N}^{(\sigma)}=0, \\
z(x, 0)=0,
\end{gathered}
$$

где $\Psi=O\left(h^{2}+\tau^{2}\right)$ - погрешности аппроксимации дифференциальной задачи (1.1)-(1.3) разностной схемой $(3.1)-(3.3)$ в классе решений $u=u(x, t)$ задачи $(1.1)-(1.3)$.

Применяя априорную оценку (3.8) к решению задачи (3.9)-(3.11), получаем неравенство

$$
\left\|z^{j+1}\right\|_{1}^{2} \leq M \max _{0 \leq j^{\prime} \leq j}\left\|\Psi^{j^{\prime}}\right\|_{0}^{2},
$$

где $M-$ положительная постоянная, не зависящая от $h$ и $\tau$.

Из априорной оценки (3.12) следует сходимость решения разностной задачи (3.1)-(3.3) к решению дифференциальной задачи (1.1)-(1.3) в смысле нормы $\left\|z^{j+1}\right\|_{1}^{2}$ на каждом слое так, что существует такое $\tau_{0}$, что при $\tau \leq \tau_{0}$ справедлива оценка

$$
\left\|y^{j+1}-u^{j+1}\right\|_{1} \leq M\left(h^{2}+\tau^{2}\right) .
$$

4. Постановка нелокальной краевой задачи и априорная оценка в дифференциальной форме. Рассмотрим теперь нелокальную краевую задачу для уравнения (1.1)

$$
\begin{aligned}
& \Pi(0, t)=\beta_{11}(t) u(0, t)+\beta_{12}(t) \partial_{0 t}^{\alpha} u(0, t)-\mu_{1}(t), \\
& -\Pi(l, t)=\beta_{21}(t) u(l, t)+\beta_{22}(t) \partial_{0 t}^{\alpha} u(l, t)-\mu_{2}(t),
\end{aligned}
$$


где

$0<c_{0} \leq k, \eta, \beta_{12}, \beta_{22} \leq c_{1}, \quad\left|\beta_{11}\right|,\left|\beta_{21}\right|,|r|,|q|,\left|\eta_{x}\right|,\left|k_{x}\right| \leq c_{2}, \quad \Pi(x, t)=k u_{x}+\partial_{0 t}^{\alpha}\left(\eta(x) u_{x}\right)$.

Умножим уравнение (1.1) скалярно на $U=u+\partial_{0 t}^{\alpha} u-u_{x x}$

$$
\left(\partial_{0 t}^{\alpha} u, U\right)=\left(\left(k u_{x}\right)_{x}, U\right)+\left(\partial_{0 t}^{\alpha}\left(\eta u_{x}\right)_{x}, U\right)+\left(r u_{x}\left(x_{0}, t\right), U\right)-(q u, U)+(f, U) .
$$

Из (4.3) после нетрудных преобразований находим [6]

$$
\begin{gathered}
\frac{1}{2} \partial_{0 t}^{\alpha}\|u\|_{0}^{2}+\left(\frac{1}{2}+c_{0}\right) \partial_{0 t}^{\alpha}\left\|u_{x}\right\|_{0}^{2}+\left\|\partial_{0 t}^{\alpha} u\right\|_{0}^{2}+c_{0}\left(\left\|u_{x}\right\|_{0}^{2}+\left\|u_{x x}\right\|_{0}^{2}\right)+\frac{c_{0}}{2}\left\|\partial_{0 t}^{\alpha} u_{x}\right\|_{0}^{2}+\frac{c_{0}}{2} \partial_{0 t}^{\alpha}\left\|u_{x x}\right\|_{0}^{2} \leq \\
\leq\left. u \Pi(x, t)\right|_{0} ^{l}+\left.u_{x} \partial_{0 t}^{\alpha} u\right|_{0} ^{l}+\left.\partial_{0 t}^{\alpha} u \Pi(x, t)\right|_{0} ^{l}+\varepsilon M_{1}\left\|\partial_{0 t}^{\alpha} u\right\|_{0}^{2}+M_{2}^{\varepsilon}\left(\|u\|_{0}^{2}+\left\|u_{x}\right\|_{0}^{2}+\left\|u_{x x}\right\|_{0}^{2}\right)+ \\
+M_{3}^{\varepsilon}\|f\|_{0}^{2}
\end{gathered}
$$

Преобразуем первое, второе и третье слагаемые в правой части (4.4)

$$
\begin{gathered}
\left.u \Pi(x, t)\right|_{0} ^{l}=u(l, t) \Pi(l, t)-u(0, t) \Pi(0, t)=u(l, t)\left(\mu_{2}(t)-\beta_{21}(t) u(l, t)-\beta_{22}(t) \partial_{0 t}^{\alpha} u(l, t)\right)+ \\
+u(0, t)\left(\mu_{1}(t)-\beta_{11}(t) u(0, t)-\beta_{12}(t) \partial_{0 t}^{\alpha} u(0, t)\right)=\mu_{2}(t) u(l, t)-\beta_{21} u^{2}(l, t)-\beta_{22}(t) u(l, t) \partial_{0 t}^{\alpha} u(l, t)+ \\
+\mu_{1}(t) u(0, t)-\beta_{11}(t) u^{2}(0, t)-\beta_{12}(t) u(0, t) \partial_{0 t}^{\alpha} u(0, t) \leq-\frac{\beta_{22}(t)}{2} \partial_{0 t}^{\alpha} u^{2}(l, t)-\frac{\beta_{12}}{2} \partial_{0 t}^{\alpha} u^{2}(0, t)+ \\
+M_{4}\left(\|u\|_{0}^{2}+\left\|u_{x}\right\|_{0}^{2}\right)+M_{5}\left(\mu_{1}^{2}(t)+\mu_{2}^{2}(t)\right), \\
+\left(\mu_{1}(t)-u_{x}(0, t)-\beta_{11}(t) u(0, t)-\beta_{12}(t) \partial_{0 t}^{\alpha} u(0, t)\right) \partial_{0 t}^{\alpha} u(0, t)=u_{x}(l, t) \partial_{0 t}^{\alpha} u(l, t)+\mu_{2}(t) \partial_{0 t}^{\alpha} u(l, t)- \\
\quad-\beta_{21}(t) u(l, t) \partial_{0 t}^{\alpha} u(l, t)-\beta_{22}(t)\left(\partial_{0 t}^{\alpha} u(l, t)\right)^{2}+\mu_{1}(t) \partial_{0 t}^{\alpha} u(0, t)-u_{x}(0, l) \partial_{0 t}^{\alpha} u(0, t)- \\
-\beta_{11}(t) u(0, t) \partial_{0 t}^{\alpha} u(0, t)-\beta_{12}(t)\left(\partial_{0 t}^{\alpha} u(0, t)\right)^{2} \leq-\beta_{22}(t)\left(\partial_{0 t}^{\alpha} u(l, t)\right)^{2}-\beta_{12}(t)\left(\partial_{0 t}^{\alpha} u(0, t)\right)^{2}+ \\
+\varepsilon_{1}\left(\partial_{0 t}^{\alpha} u(0, t)\right)^{2}+\varepsilon_{2}\left(\partial_{0 t}^{\alpha} u(l, t)\right)^{2}+M_{6}\left(\|u\|_{0}^{2}+\left\|u_{x}\right\|_{0}^{2}+\left\|u_{x x}\right\|_{0}^{2}\right)+M_{7}\left(\mu_{1}^{2}(t)+\mu_{2}^{2}(t)\right) \leq \\
-\frac{\beta_{22}}{2}\left(\partial_{0 t}^{\alpha} u(l, t)\right)^{2}-\frac{\beta_{12}}{2}\left(\partial_{0 t}^{\alpha} u(0, t)\right)^{2}+M_{6}\left(\|u\|_{0}^{2}+\left\|u_{x}\right\|_{0}^{2}+\left\|u_{x x}\right\|_{0}^{2}\right)+M_{7}\left(\mu_{1}^{2}(t)+\mu_{2}^{2}(t)\right) . \quad(4.6)
\end{gathered}
$$

Учитывая преобразования $(4.5),(4.6)$, из (4.4) при $\varepsilon=\frac{1}{2 M_{1}}$ находим

$$
\partial_{0 t}^{\alpha}\|u\|_{W_{2}^{2}(0, l)}^{2}+\left\|u_{x}\right\|_{0}^{2}+\left\|u_{x x}\right\|_{0}^{2}+\left\|\partial_{0 t}^{\alpha} u\right\|_{0}^{2}+\left\|\partial_{0 t}^{\alpha} u_{x}\right\|_{0}^{2} \leq M_{8}\|u\|_{W_{2}^{2}(0, l)}^{2}+M_{9}\left(\|f\|_{0}^{2}+\mu_{1}^{2}+\mu_{2}^{2}\right),
$$

где $\|u\|_{W_{2}^{2}(0, l)}^{2}=\|u\|_{0}^{2}+\left\|u_{x}\right\|_{0}^{2}+\left\|u_{x x}\right\|_{0}^{2}$.

Применяя к обеим частям неравенства (4.7) оператор дробного интегрирования $D_{0 t}^{-\alpha}$, на основании леммы 2 [8] получаем априорную оценку

$$
\|u\|_{3}^{2} \leq M_{10}\left(D_{0 t}^{-\alpha}\left(\|f\|_{0}^{2}+\mu_{1}^{2}+\mu_{2}^{2}\right)+\left\|u_{0}\right\|_{W_{2}^{2}(0, l)}^{2}\right),
$$

где $\|u\|_{3}^{2}=\|u\|_{W_{2}^{2}(0, l)}^{2}+D_{0 t}^{-\alpha}\left(\left\|u_{x}\right\|_{0}^{2}+\left\|u_{x x}\right\|_{0}^{2}+\left\|\partial_{0 t}^{\alpha} u\right\|_{0}^{2}+\left\|\partial_{0 t}^{\alpha} u_{x}\right\|_{0}^{2}\right), M_{10}$ - положительная постоянная, зависящая только от входных данных задачи (1.1), (4.1), (1.3), $D_{0 t}^{-\alpha} u=\frac{1}{\Gamma(\alpha)} \int_{0}^{t} \frac{u d \tau}{(t-\tau)^{1-\alpha}}$ - дробный интеграл Римана-Лиувилля порядка $\alpha, 0<\alpha<1$.

Из априорной оценки (4.8) следуют единственность и устойчивость решения по начальным данным и правой части в смысле нормы $\|u\|_{3}^{2}$. 
Теорема 3. Если $k(x, t) \in C^{1,0}\left(\bar{Q}_{T}\right), \eta(x) \in C^{1}[0, l], q(x, t), f(x, t) \in C\left(\bar{Q}_{T}\right), u(x, t) \in$ $C^{(2,0)}\left(Q_{T}\right) \cap C^{(1,0)}\left(\bar{Q}_{T}\right), \partial_{0 t}^{\alpha} u(x, t) \in C\left(\bar{Q}_{T}\right)$ и выполнены условия (1.4), (4.2), то для решения задачи (1.1), (4.1), (1.3) справедлива априорная оценка (4.8).

5. Устойчивость и сходимость разностной схемы. На равномерной сетке $\bar{\omega}_{h \tau}$ дифференциальной задаче $(1.1),(4.1),(1.3)$ поставим в соответствие разностную схему порядка аппроксимации $O\left(h^{2}+\tau^{2}\right)$

$$
\begin{gathered}
\Delta_{0 t_{j+\sigma}}^{\alpha} y_{i}=\left(a^{j} y_{\bar{x}}^{(\sigma)}\right)_{x, i}+\Delta_{0 t_{j+\sigma}}^{\alpha}\left(\gamma y_{\bar{x}}\right)_{x, i}+r_{i}^{j}\left(y_{x, i_{0}}^{(\sigma)} x_{i_{0}}^{-}+y_{\dot{x}, i_{0}+1}^{(\sigma)} x_{i_{0}}^{+}\right)-d_{i}^{j} y_{i}^{(\sigma)}+\varphi_{i}^{j}, \\
a_{1} y_{x, 0}^{(\sigma)}+\Delta_{0 t_{j+\sigma}}^{\alpha}\left(\gamma_{1} y_{\bar{x}, 0}\right)+0.5 h r_{0}\left(y_{\tilde{x}, i_{0}}^{(\sigma)} x_{i_{0}}^{-}+y_{\tilde{x}, i_{0}+1}^{(\sigma)} x_{i_{0}}^{+}\right)= \\
=\widetilde{\beta}_{11} y_{0}^{(\sigma)}-\widetilde{\mu}_{1}+\widetilde{\beta}_{12} \Delta_{0 t_{j+\sigma}}^{\alpha} y_{0}, \quad t \in \bar{\omega}_{\tau}, x=0, \\
-\left(a_{N} y_{\bar{x}, N}^{(\sigma)}-\Delta_{0 t_{j+\sigma}}^{\alpha}\left(\gamma_{N} y_{\bar{x}, N}\right)\right)+0.5 h r_{N}\left(y_{\dot{x}, i_{0}}^{(\sigma)} x_{i_{0}}^{-}+y_{\dot{x}, i_{0}+1}^{(\sigma)} x_{i_{0}}^{+}\right)= \\
=\widetilde{\beta}_{21} y_{N}^{(\sigma)}-\widetilde{\mu}_{2}+\widetilde{\beta}_{22} \Delta_{0 t_{j+\sigma}}^{\alpha} y_{N}, \quad t \in \bar{\omega}_{\tau}, \quad x=l, \\
y(x, 0)=u_{0}(x), \quad x \in \bar{\omega}_{h},
\end{gathered}
$$

где

$\widetilde{\beta}_{11}\left(t_{j+\sigma}\right)=\beta_{11}\left(t_{j+\sigma}\right)+0.5 h d_{0}^{j}, \quad \widetilde{\beta}_{12}\left(t_{j+\sigma}\right)=\beta_{12}\left(t_{j+\sigma}\right)+0.5 h, \widetilde{\mu}_{1}\left(t_{j+\sigma}\right)=\mu_{1}\left(t_{j+\sigma}\right)+0.5 h \varphi_{0}^{j}$, $\widetilde{\beta}_{21}\left(t_{j+\sigma}\right)=\beta_{21}\left(t_{j+\sigma}\right)+0.5 h d_{N}^{j}, \quad \widetilde{\beta_{22}}\left(t_{j+\sigma}\right)=\beta_{22}\left(t_{j+\sigma}\right)+0.5 h, \widetilde{\mu}_{2}\left(t_{j+\sigma}\right)=\mu_{2}\left(t_{j+\sigma}\right)+0.5 h \varphi_{N}^{j}$.

Найдем априорную оценку методом энергетических неравенств, для этого умножим уравнение (5.1) скалярно на $\bar{y}=y^{(\sigma)}+\Delta_{0 t_{j+\sigma}}^{\alpha} y-y_{\bar{x} x}^{(\sigma)}$

$$
\begin{aligned}
\left(\Delta_{0 t_{j+\sigma}}^{\alpha} y, \bar{y}\right)=\left(\left(a y_{\bar{x}}^{(\sigma)}\right)_{x}, \bar{y}\right)+ & \left(\Delta_{0 t_{j+\sigma}}^{\alpha}\left(\gamma_{i} y_{\bar{x}}\right)_{x}, \bar{y}\right)+\left(r_{i}^{j}\left(y_{\dot{x}, i_{0}}^{(\sigma)} x_{i_{0}}^{-}+y_{\dot{x}, i_{0}+1}^{(\sigma)} x_{i_{0}}^{+}\right), \bar{y}\right)- \\
& -\left(d y^{(\sigma)}, \bar{y}\right)+(\varphi, \bar{y}),
\end{aligned}
$$

где $[u, v]=\sum_{i=0}^{N} u_{i} v_{i} \hbar, \hbar=\left\{\begin{array}{ll}0.5 h, & i=0, N ; \\ h, & i \neq 0, N,\end{array}[u, u]=\left[1, u^{2}\right]=|[u]|_{0}^{2},(u, v]=\sum_{i=1}^{N-1} u_{i} v_{i} h\right.$.

Из (5.5) после некоторых преобразований получим

$$
\begin{gathered}
\left.\left.\left.\left.\left(\Delta_{0 t_{j+\sigma}}^{\alpha} y, \bar{y}\right)+c_{0} \| y_{\bar{x}}^{(\sigma)}\right]\left.\right|_{0} ^{2}+\frac{c_{0}}{2} \Delta_{0 t_{j+\sigma}}^{\alpha} \| y_{\bar{x}}\right]\left.\right|_{0} ^{2}+c_{0}\left\|y_{\bar{x} x}^{(\sigma)}\right\|_{0}^{2}+\frac{c_{0}}{2} \Delta_{0 t_{j+\sigma}}^{\alpha} \| y_{\bar{x}}\right]\left.\right|_{0} ^{2}+\frac{c_{0}}{2} \| \Delta_{0 t_{j+\sigma}}^{\alpha} y_{\bar{x}}\right]\left.\right|_{0} ^{2}+ \\
\left.+\frac{c_{0}}{2} \Delta_{0 t_{j+\sigma}}^{\alpha}\left\|y_{\bar{x} x}\right\|_{0}^{2} \leq\left.\left(a y_{\bar{x}}^{(\sigma)}+\Delta_{0 t_{j+\sigma}}^{\alpha}\left(\gamma y_{\bar{x}}\right)\right)\left(y^{(\sigma)}+\Delta_{0 t_{j+\sigma}}^{\alpha} y\right)\right|_{0} ^{N}+\left.M_{1}\left(\| y_{\bar{x}}^{(\sigma)}\right]\right|_{0} ^{2}+\left\|y_{\bar{x} x}^{(\sigma)}\right\|_{0}^{2}\right)+ \\
+\left(r\left(y_{\dot{x}, i_{0}}^{(\sigma)} \frac{x_{i_{0}+1}-x_{0}}{h}+y_{\dot{x}, i_{0}+1}^{(\sigma)} \frac{x_{0}-x_{i_{0}}}{h}\right), \bar{y}\right)-\left(d y^{(\sigma)}, \bar{y}\right)+(\varphi, \bar{y}) .
\end{gathered}
$$

Преобразуем первое слагаемое в правой части (5.6)

$$
\begin{gathered}
\left.\left(a y_{\bar{x}}^{(\sigma)}+\Delta_{0 t_{j+\sigma}}^{\alpha}\left(\gamma y_{\bar{x}}\right)\right)\left(y^{(\sigma)}+\Delta_{0 t_{j+\sigma}}^{\alpha} y\right)\right|_{0} ^{N}=\left(a_{N} y_{\bar{x}, N}^{(\sigma)}+\Delta_{0 t_{j+\sigma}}^{\alpha} \gamma_{N} y_{\bar{x}, N}\right)\left(y_{N}^{(\sigma)}+\Delta_{0 t_{j+\sigma}}^{\alpha} y_{N}\right)- \\
-\left(a_{1} y_{x, 0}^{(\sigma)}+\Delta_{0 t_{j+\sigma}}^{\alpha} \gamma_{1} y_{x, 0}\right)\left(y_{0}^{(\sigma)}+\Delta_{0 t_{j+\sigma}}^{\alpha} y_{0}\right)=\left[\widetilde{\mu}_{2}-\widetilde{\beta}_{21} y_{N}^{(\sigma)}-\widetilde{\beta}_{22} \Delta_{0 t_{j+\sigma}}^{\alpha} y_{N}+0.5 h r_{N}\left(y_{x, i_{0}}^{(\sigma)} \frac{x_{i_{0}+1}-x_{0}}{h}+\right.\right. \\
\left.\left.+y_{\tilde{x}, i_{0}+1}^{(\sigma)} \frac{x_{0}-x_{i_{0}}}{h}\right)\right]\left(y_{N}^{(\sigma)}+\Delta_{0 t_{j+\sigma}}^{\alpha} y_{N}\right)+\left[\widetilde{\mu}_{1}-\widetilde{\beta}_{11} y_{0}^{(\sigma)}-\widetilde{\beta}_{22} \Delta_{0 t_{j+\sigma}}^{\alpha} y_{0}+0.5 h r_{0}\left(y_{x, i_{0}}^{(\sigma)} \frac{x_{i_{0}+1}-x_{0}}{h}+\right.\right. \\
\left.\left.+y_{\grave{x}, i_{0}+1}^{(\sigma)} \frac{x_{0}-x_{i_{0}}}{h}\right)\right]\left(y_{0}^{(\sigma)}+\Delta_{0 t_{j+\sigma}}^{\alpha} y_{0}\right)=\left(\mu_{2}-\beta_{21} y_{N}^{(\sigma)}-\beta_{22} \Delta_{0 t_{j+\sigma}}^{\alpha} y_{N}\right)\left(y_{N}^{(\sigma)}+\Delta_{0 t_{j+\sigma}}^{\alpha} y_{N}\right)+ \\
+0.5 h\left(\varphi_{N}-d_{N} y_{N}^{(\sigma)}-\Delta_{0 t_{j+\sigma}}^{\alpha} y_{N}+r_{N}\left(y_{\dot{x}, i_{0}}^{(\sigma)} \frac{x_{i_{0}+1}-x_{0}}{h}+y_{\grave{x}, i_{0}+1}^{(\sigma)} \frac{x_{0}-x_{i_{0}}}{h}\right)\right)\left(y_{N}^{(\sigma)}+\Delta_{0 t_{j+\sigma}}^{\alpha} y_{N}\right)+ \\
\quad+\left(\mu_{1}-\beta_{11} y_{0}^{(\sigma)}-\beta_{12} \Delta_{0 t_{j+\sigma}}^{\alpha} y_{0}\right)\left(y_{0}^{(\sigma)}+\Delta_{0 t_{j+\sigma}}^{\alpha} y_{0}\right)+0.5 h\left[\varphi_{0}-d_{0} y_{0}^{(\sigma)}-\Delta_{0 t_{j+\sigma}}^{\alpha} y_{0}+\right.
\end{gathered}
$$




$$
\left.+r_{0}\left(y_{\dot{x}, i_{0}}^{(\sigma)} \frac{x_{i_{0}+1}-x_{0}}{h}+y_{\dot{x}, i_{0}+1}^{(\sigma)} \frac{x_{0}-x_{i_{0}}}{h}\right)\right]\left(y_{0}^{(\sigma)}+\Delta_{0 t_{j+\sigma}}^{\alpha} y_{0}\right) .
$$

Преобразуем первое и третье слагаемые в правой части (5.7)

$$
\begin{gathered}
\left(\mu_{2}-\beta_{21} y_{N}^{(\sigma)}-\beta_{22} \Delta_{0 t_{j+\sigma}}^{\alpha} y_{N}\right)\left(y_{N}^{(\sigma)}+\Delta_{0 t_{j+\sigma}}^{\alpha} y_{N}\right)+\left(\mu_{1}-\beta_{11} y_{0}^{(\sigma)}-\beta_{12} \Delta_{0 t_{j+\sigma}}^{\alpha} y_{0}\right)\left(y_{0}^{(\sigma)}+\Delta_{0 t_{j+\sigma}}^{\alpha} y_{0}\right)= \\
=\mu_{1} y_{0}^{(\sigma)}+\mu_{2} y_{N}^{(\sigma)}+\mu_{1} \Delta_{0 t_{j+\sigma}}^{\alpha} y_{0}+\mu_{2} \Delta_{0 t_{j+\sigma}}^{\alpha} y_{N}-\beta_{21}\left(y_{N}^{(\sigma)}\right)^{2}-\beta_{11}\left(y_{0}^{(\sigma)}\right)^{2}-\beta_{21} y_{N}^{(\sigma)} \Delta_{0 t_{j+\sigma}}^{\alpha} y_{N}- \\
-\beta_{11} y_{0}^{(\sigma)} \Delta_{0 t_{j+\sigma}}^{\alpha} y_{0}-\beta_{22} y_{N}^{(\sigma)} \Delta_{0 t_{j+\sigma}}^{\alpha} y_{N}-\beta_{12} y_{0}^{(\sigma)} \Delta_{0 t_{j+\sigma}}^{\alpha} y_{0}-\beta_{22}\left(\Delta_{0 t_{j+\sigma}}^{\alpha} y_{N}\right)^{2}-\beta_{12}\left(\Delta_{0 t_{j+\sigma}}^{\alpha} y_{0}\right)^{2} \leq \\
\leq-\beta_{22}\left(\Delta_{0 t_{j+\sigma}}^{\alpha} y_{N}\right)^{2}-\beta_{12}\left(\Delta_{0 t_{j+\sigma}}^{\alpha} y_{0}\right)^{2}-\frac{\beta_{22}}{2} \Delta_{0 t_{j+\sigma}}^{\alpha} y_{N}^{2}-\frac{\beta_{12}}{2} \Delta_{0 t_{j+\sigma}}^{\alpha} y_{0}^{2}+\varepsilon_{1}\left(\Delta_{0 t_{j+\sigma}}^{\alpha} y_{0}\right)^{2}+ \\
\left.+\varepsilon_{2}\left(\Delta_{0 t_{j+\sigma}}^{\alpha} y_{N}\right)^{2}+\left.M_{2}^{\varepsilon}\left(\left|\left[y^{(\sigma)}\right]\right|_{0}^{2}+\| y_{\bar{x}}^{(\sigma)}\right]\right|_{0} ^{2}\right)+M_{3}^{\varepsilon}\left(\mu_{1}^{2}+\mu_{2}^{2}\right) \leq-\frac{\beta_{22}}{2}\left(\Delta_{0 t_{j+\sigma}}^{\alpha} y_{N}\right)^{2}-\frac{\beta_{12}}{2}\left(\Delta_{0 t_{j+\sigma}}^{\alpha} y_{0}\right)^{2}- \\
\left.\quad-\frac{\beta_{22}}{2} \Delta_{0 t_{j+\sigma}}^{\alpha} y_{N}^{2}-\frac{\beta_{12}}{2} \Delta_{0 t_{j+\sigma}}^{\alpha} y_{0}^{2}+\left.M_{4}\left(\left|\left[y^{(\sigma)}\right]\right|_{0}^{2}+\| y_{\bar{x}}^{(\sigma)}\right]\right|_{0} ^{2}\right)+M_{5}\left(\mu_{1}^{2}+\mu_{2}^{2}\right) .
\end{gathered}
$$

Учитывая (5.7) и (5.8), из (5.6) находим

$$
\begin{gathered}
\left.\left.\left.\quad\left[\Delta_{0 t_{j+\sigma}}^{\alpha} y, y^{(\sigma)}+\Delta_{0 t_{j+\sigma}}^{\alpha} y\right]+c_{0} \| y_{\bar{x}}^{(\sigma)}\right]\left.\right|_{0} ^{2}+\frac{c_{0}}{2} \Delta_{0 t_{j+\sigma}}^{\alpha} \| y_{\bar{x}}\right]\left.\right|_{0} ^{2}+c_{0}\left\|y_{\bar{x} x}^{(\sigma)}\right\|_{0}^{2}+\frac{c_{0}}{2} \Delta_{0 t_{j+\sigma}}^{\alpha} \| y_{\bar{x}}\right]\left.\right|_{0} ^{2}+ \\
\left.+\frac{c_{0}}{2} \| \Delta_{0 t_{j+\sigma}}^{\alpha} y_{\bar{x}}\right]\left.\right|_{0} ^{2}+\frac{c_{0}}{2} \Delta_{0 t_{j+\sigma}}^{\alpha}\left\|y_{\bar{x} x}\right\|_{0}^{2}+\frac{\beta_{22}}{2}\left(\Delta_{0 t_{j+\sigma}}^{\alpha} y_{N}\right)^{2}+\frac{\beta_{12}}{2}\left(\Delta_{0 t_{j+\sigma}}^{\alpha} y_{0}\right)^{2}+\frac{\beta_{22}}{2} \Delta_{0 t_{j+\sigma}}^{\alpha} y_{N}^{2}+ \\
\left.+\frac{\beta_{12}}{2} \Delta_{0 t_{j+\sigma}}^{\alpha} y_{0}^{2} \leq\left(\Delta_{0 t_{j+\sigma}}^{\alpha} y, y_{\bar{x} x}^{(\sigma)}\right)+\left.M_{6}\left(\left|\left[y^{(\sigma)}\right]\right|_{0}^{2}+\| y_{\bar{x}}^{(\sigma)}\right]\right|_{0} ^{2}+\left\|y_{\bar{x} x}^{(\sigma)}\right\|_{0}^{2}\right)+M_{7}\left(\mu_{1}^{2}+\mu_{2}^{2}\right)+ \\
\quad+\left[r\left(y_{\dot{x}, i_{0}}^{(\sigma)} \frac{x_{i_{0}+1}-x_{0}}{h}+y_{\dot{x}, i_{0}+1}^{(\sigma)} \frac{x_{0}-x_{i_{0}}}{h}\right), y^{(\sigma)}+\Delta_{0 t_{j+\sigma}}^{\alpha} y\right]-\left[d y^{(\sigma)}, y^{(\sigma)}+\Delta_{0 t_{j+\sigma}}^{\alpha} y\right]+ \\
+\left[\varphi, y^{(\sigma)}+\Delta_{0 t_{j+\sigma}}^{\alpha} y\right]-\left(r\left(y_{\dot{x}, i_{0}}^{(\sigma)} \frac{x_{i_{0}+1}-x_{0}}{h}+y_{\dot{x}, i_{0}+1}^{(\sigma)} \frac{x_{0}-x_{i_{0}}}{h}\right), y_{\bar{x} x}^{\sigma}\right)+\left(d y^{(\sigma)}, y_{\bar{x} x}^{(\sigma)}\right)+\left(\varphi, y_{\bar{x} x}^{(\sigma)}\right) .
\end{gathered}
$$

Из (5.9) после несложных преобразований получим

$$
\begin{gathered}
\left.\left.\left.\frac{1}{2} \Delta_{0 t_{j+\sigma}}^{\alpha}|[y]|^{2}+\left|\left[\Delta_{0 t_{j+\sigma}}^{\alpha} y\right]\right|_{0}^{2}+\left(c_{0}+\frac{1}{2}\right) \Delta_{0 t_{j+\sigma}}^{\alpha} \| y_{\bar{x}}\right]\left.\right|_{0} ^{2}+\frac{c_{0}}{2} \Delta_{0 t_{j+\sigma}}^{\alpha}\left\|y_{\bar{x} x}\right\|_{0}^{2}+\frac{c_{0}}{2} \| \Delta_{0 t_{j+\sigma}}^{\alpha} y_{\bar{x}}\right]\left.\right|_{0} ^{2}+c_{0} \| y_{\bar{x}}^{(\sigma)}\right]\left.\right|_{0} ^{2}+ \\
+c_{0}\left\|y_{\bar{x} x}^{(\sigma)}\right\|_{0}^{2}+\frac{\beta_{22}}{2}\left(\Delta_{0 t_{j+\sigma}}^{\alpha} y_{N}\right)^{2}+\frac{\beta_{12}}{2}\left(\Delta_{0 t_{j+\sigma}}^{\alpha} y_{0}\right)^{2}+\frac{\beta_{22}}{2} \Delta_{0 t_{j+\sigma}}^{\alpha} y_{N}^{2}+\frac{\beta_{12}}{2} \Delta_{0 t_{j+\sigma}}^{\alpha} y_{0}^{2} \leq \\
\left.\leq \varepsilon_{1}\left(\Delta_{0 t_{j+\sigma}}^{\alpha} y_{N}\right)^{2}+\varepsilon_{2}\left(\Delta_{0 t_{j+\sigma}}^{\alpha} y_{0}\right)^{2}+M_{8} \varepsilon\left|\left[\Delta_{0 t_{j+\sigma}}^{\alpha} y\right]\right|_{0}^{2}+\left.M_{9}^{\varepsilon}\left(\left|\left[y^{(\sigma)}\right]\right|_{0}^{2}+\| y_{\bar{x}}^{(\sigma)}\right]\right|_{0} ^{2}+\left\|y_{\bar{x} x}^{(\sigma)}\right\|_{0}^{2}\right)+ \\
+M_{10}^{\varepsilon}\left(|[\varphi]|+\mu_{1}^{2}+\mu_{2}^{2}\right) .
\end{gathered}
$$

Выбирая $\varepsilon_{1}=\frac{\beta_{22}}{2}, \varepsilon_{2}=\frac{\beta_{12}}{2}, \varepsilon=\frac{1}{2 M_{8}}$, из (5.10) находим

$$
\begin{gathered}
\left.\left.\Delta_{0 t_{j+\sigma}}^{\alpha}|[y]|_{W_{2}^{2}(0, l)}^{2}+\| y_{\bar{x}}^{(\sigma)}\right]\left.\right|_{0} ^{2}+\left\|y_{\bar{x} x}^{(\sigma)}\right\|_{0}^{2}+\| \Delta_{0 t_{j+\sigma}}^{\alpha} y \bar{x}\right]\left.\right|_{0} ^{2}+\left|\left[\Delta_{0 t_{j+\sigma}}^{\alpha} y\right]\right|_{0}^{2} \leq M_{11}\left|\left[y^{(\sigma)}\right]\right|_{W_{2}^{2}(0, l)}^{2}+ \\
+M_{12}\left(|[\varphi]|_{0}^{2}+\mu_{1}^{2}+\mu_{2}^{2}\right)
\end{gathered}
$$

где $\left.|[y]|_{W_{2}^{2}(0, l)}^{2}=|[y]|_{0}^{2}+\| y \bar{x}\right]\left.\right|_{0} ^{2}+\left\|y_{\bar{x} x}\right\|_{0}^{2}$.

Перепишем (5.11) в другой форме

$$
\Delta_{0 t_{j+\sigma}}^{\alpha}\left|\left[y^{j}\right]\right|_{W_{2}^{2}(0, l)}^{2} \leq M_{13}^{\sigma}\left|\left[y^{j+1}\right]\right|_{W_{2}^{2}(0, l)}^{2}+M_{14}^{\sigma}\left|\left[y^{j}\right]\right|_{W_{2}^{2}(0, l)}^{2}+M_{12}\left(|[\varphi]|_{0}^{2}+\mu_{1}^{2}+\mu_{2}^{2}\right) .
$$

На основании леммы 7 [6] из (5.12) находим априорную оценку

$$
\left|\left[y^{j+1}\right]\right|_{W_{2}^{2}(0, l)}^{2} \leq M_{15}\left(\left|\left[y^{0}\right]\right|_{W_{2}^{2}(0, l)}^{2}+\frac{t_{j}^{\alpha}}{\Gamma(1+\alpha)} \max _{0 \leq j^{\prime} \leq j}\left(\left|\left[\varphi^{j^{\prime}}\right]\right|_{0}^{2}+\mu_{1}^{2}+\mu_{2}^{2}\right)\right),
$$

где $M_{15}$ - положительная постоянная, не зависящая от $h$ и $\tau$. 
Из априорной оценки (5.13) следуют единственность и устойчивость решения задачи (5.1)-(5.4) по начальным данным и правой части в смысле нормы $\left|\left[y^{j+1}\right]\right|_{W_{2}^{2}(0, l)}^{2}$.

Теорема 4. Пусть выполнены условия (1.4), (4.2), тогда существует такое малое $\tau_{0}=$ $\tau_{0}\left(c_{0}, c_{1}, c_{2}, \alpha, \sigma\right)$, что если $\tau \leq \tau_{0}$, то для решения разностной задачи (5.1)-(5.4) справедлива априорная оченка (5.13).

Пусть $u(x, t)$ - решение задачи $(1.1),(4.1),(1.3), y\left(x_{i}, t_{j}\right)=y_{i}^{j}-$ решение разностной задачи (5.1)-(5.4). Для оценки точности разностной схемы (5.1)-(5.4) рассмотрим разность $z_{i}^{j}=y_{i}^{j}-u_{i}^{j}$, где $u_{i}^{j}=u\left(x_{i}, t_{j}\right)$. Тогда, подставляя $y=z+u$ в соотношения (5.1)-(5.4), получаем задачу для функции $z$

$$
\begin{aligned}
& \Delta_{0 t_{j+\sigma}}^{\alpha} z=\left(a^{j} z_{\bar{x}}^{(\sigma)}\right)_{x}+\Delta_{0 t_{j+\sigma}}^{\alpha}\left(\gamma_{i} z_{\bar{x}}\right)_{x}+r_{i}^{j}\left(z_{\dot{x}, i_{0}}^{(\sigma)} x_{i_{0}}^{-}+z_{\dot{x}, i_{0}+1}^{(\sigma)} x_{i_{0}}^{+}\right)-d_{i}^{j} z_{i}^{(\sigma)}+\Psi_{i}^{j}, \\
& a_{1} z_{x, 0}^{(\sigma)}+\Delta_{0 t_{j+\sigma}}^{\alpha}\left(\gamma_{1} z_{\bar{x}, 0}\right)+0.5 h r_{0}\left(z_{\dot{x}, i_{0}}^{(\sigma)} x_{i_{0}}^{-}+z_{\dot{x}, i_{0}+1}^{(\sigma)} x_{i_{0}}^{+}\right)= \\
& =\widetilde{\beta}_{11} z_{0}^{(\sigma)}+\widetilde{\beta}_{12} \Delta_{0 t_{j+\sigma}}^{\alpha} z_{0}-\widetilde{\nu}_{1}, t \in \bar{\omega}_{\tau}, \quad x=0, \\
& -\left(a_{N} z_{\bar{x}, N}^{(\sigma)}-\Delta_{0 t_{j+\sigma}}^{\alpha}\left(\gamma_{N} z_{\bar{x}, N}\right)\right)+0.5 h r_{N}\left(z_{\dot{x}, i_{0}}^{(\sigma)} x_{i_{0}}^{-}+z_{\dot{x}, i_{0}+1}^{(\sigma)} x_{i_{0}}^{+}\right)= \\
& =\widetilde{\beta}_{21} z_{N}^{(\sigma)}+\widetilde{\beta}_{22} \Delta_{0 t_{j+\sigma}}^{\alpha} z_{N}-\widetilde{\nu}_{2}, t \in \bar{\omega}_{\tau}, \quad x=l, \\
& z(x, 0)=0, \quad x \in \bar{\omega}_{h},
\end{aligned}
$$

где $\Psi=O\left(h^{2}+\tau^{2}\right), \nu_{1}=O\left(h^{2}+\tau^{2}\right), \nu_{2}=O\left(h^{2}+\tau^{2}\right)$ - погрешности аппроксимации дифференциальной задачи $(1.1),(4.1),(1.4)$ разностной схемой (5.1)-(5.4) в классе решений $u=u(x, t)$ задачи $(1.1),(4.1),(1.4)$.

Применяя априорную оценку (5.13) к решению задачи (5.14) - (5.17), получаем неравенство

$$
\left|\left[z^{j+1}\right]\right|_{W_{2}^{2}(0, l)}^{2} \leq M \max _{0 \leq j^{\prime} \leq j}\left(\left|\left[\Psi^{j^{\prime}}\right]\right|_{0}^{2}+\nu_{1}^{j^{\prime} 2}+\nu_{2}^{j^{\prime} 2}\right),
$$

где $M-$ положительная постоянная, не зависящая от $h$ и $\tau$.

Из априорной оценки (5.18) следует сходимость решения разностной задачи (5.1)-(5.4) к решению дифференциальной задачи $(1.1),(4.1),(1.4)$ в смысле нормы $\left|\left[z^{j+1}\right]\right|_{W_{2}^{2}(0, l)}^{2}$ на каждом слое так, что существует такое $\tau_{0}$, что при $\tau \leq \tau_{0}$ справедлива оценка

$$
\left|\left[y^{j+1}-u^{j+1}\right]\right|_{W_{2}^{2}(0, l)} \leq M\left(h^{2}+\tau^{2}\right) .
$$

6. Результаты численных расчетов. Для численного решения разностных схем, полученных при аппроксимации рассматриваемых в данной работе краевых задач для нагруженного псевдопараболического уравнения с дробной производной Герасимова-Капуто порядка $\alpha$, удобно использовать метод параметрической прогонки ([11], с. 131).

Рассмотрим следующий тестовый пример

$$
\begin{gathered}
\partial_{0 t}^{\alpha} u=\frac{\partial}{\partial x}\left(k(x, t) \frac{\partial u}{\partial x}\right)+\partial_{0 t}^{\alpha} \frac{\partial}{\partial x}\left(\eta(x) \frac{\partial u}{\partial x}\right)+r(x, t) \frac{\partial u}{\partial x}\left(x_{0}, t\right)-q(x, t) u+f(x, t), \\
\Pi(0, t)=\beta_{11}(t) u(0, t)+\beta_{12}(t) \partial_{0 t}^{\alpha} u(0, t)-\mu_{1}(t), \\
-\Pi(l, t)=\beta_{21}(t) u(l, t)+\beta_{22}(t) \partial_{0 t}^{\alpha} u(l, t)-\mu_{2}(t), \\
u(x, 0)=u_{0}(x), \quad 0 \leq x \leq l,
\end{gathered}
$$

где $k(x, t)=e^{x+t}, \quad \eta(x)=e^{x}, \quad r(x, t)=(x-0.5) \cos (x+t), \quad q(x, t)=\cos (x-t)$,

$$
f(x, t)=e^{x} \frac{6 t^{3-\alpha}}{\Gamma(4-\alpha)}-2 t^{3} e^{2 x+t}-2 e^{2 x}\left(\frac{6 t^{3-\alpha}}{\Gamma(4-\alpha)}+\frac{24 t^{4-\alpha}}{\Gamma(5-\alpha)}\right)
$$




$$
\begin{gathered}
-t^{3} e^{x_{0}}(x-0.5) \cos (x+t)+t^{3} e^{x} \cos (x-t), \\
\beta_{11}=0.5 e^{t}, \quad \beta_{21}=e^{l+t}, \quad \beta_{21}=\cos (t), \quad \beta_{22}=\sin (t), \\
\mu_{1}=(\cos (t)-1) \frac{6 t^{3-\alpha}}{\Gamma(4-\alpha)}-\frac{24 t^{4-\alpha}}{\Gamma(5-\alpha)}-0.5 t^{3} e^{t}, \\
\mu_{2}=2 t^{3} e^{2 l+t}+e^{l}\left(\sin (t)+e^{l}\right) \frac{6 t^{3-\alpha}}{\Gamma(4-\alpha)}+e^{2 l} \frac{24 t^{4-\alpha}}{\Gamma(5-\alpha)}, \\
u_{0}(x)=0, \quad 0 \leq x_{0} \leq 1, \quad l=1, \quad T=1 .
\end{gathered}
$$

Точное решение задачи $u(x, t)=t^{3} e^{x}$.

На данном тестовом примере при различных значениях параметров $\alpha=0.01 ; 0.5 ; 0.99$, $x_{0}=0.1 ; 0.5 ; 0.9$ и уменьшении размера сетки были исследованы максимальное значение погрешности $(z=y-u)$ и порядок сходимости $(П С)$ в нормах $|[\cdot]|_{0}$ и $\|\cdot\|_{C\left(\bar{w}_{h \tau}\right)}$, где $\|y\|_{C\left(\bar{w}_{h \tau}\right)}=\max _{\left(x_{i}, t_{j}\right) \in \bar{w}_{h \tau}}|y|$, когда $h=\tau$. Полученные результаты показывают, что погрешность уменьшается в соответствии с порядком аппроксимации $O\left(h^{2}+\tau^{2}\right)$.

Порядок сходимости определялся по следующей формуле:

$$
\Pi C=\log _{\frac{h_{1}}{h_{2}}} \frac{\left|\left[z_{1}\right]\right|_{0}}{\left|\left[z_{2}\right]\right|_{0}}=\frac{\ln \left(\frac{\left|\left[z_{1}\right]\right|_{0}}{\left|\left[z_{2}\right]\right|_{0}}\right)}{\ln \left(\frac{\left|\left[N_{2}\right]\right|_{0}}{\left|\left[N_{1}\right]\right|_{0}}\right)},
$$

где $z_{i}$ - это погрешность, соответствующая $h_{i}$.

Заключение. Настоящая работа посвящена изучению локальных и нелокальных краевых задач для одномерного по пространству нагруженного дифференциального уравнения в частных производных с переменными коэффициентами и с дробной производной Герасимова-Капуто, а также разностных схем, аппроксимирующих эти задачи на равномерных сетках. Рассмотрен классический случай нагруженного уравнения, в котором коэффициенты при старших производных отделены от нуля положительной константой. Для решения локальных и нелокальных краевых задач получены априорные оценки в дифференциальной и разностной трактовках, из чего следуют единственность и устойчивость решения по начальным данным и правой части, а также сходимость решения разностной задачи к решению дифференциальной задачи со скоростью $O\left(h^{2}+\tau^{2}\right)$. Проведены численные эксперименты, подтверждающие достоверность полученных результатов в работе.

Считаю своим приятным долгом выразить искреннюю и глубокую благодарность моему учителю Мухамеду Хабаловичу Шханукову-Лафишеву.

\section{ЛитеРАТУРА}

[1] Нахушев А.М. Нагруженные уравнения и их приложения, Дифференц. уравнения 19 (1), 86-94 (1983).

[2] Канчукоев В.З. Краевые задачи для уравнения третьего порядка смешанного гиперболопсевдопараболического типа, Дисс. ... канд. физ.-матем. наук - М., 1984. - 101 с.

[3] Канчукоев В.З. Краевые задачи для уравнений псевдопараболического и смешанного гиперболопсевдопараболического типов и их приложения $к$ расчету тепломассобмена в почвогрунтах (В сб.: САПР и АСПР в мелиорации, Нальчик, 1983).

[4] Кочина Н.И. Вопросъ регулирования уровня грунтовых вод при поливах, ДАН СССР 213 (1), 51-54 (1973).

[5] Нахушев А.М., Борисов В.Н. Краевые задачи для нагруженных параболических уравнений и их приложения к прогнозу уровня грунтовых вод, Дифференц. уравнения 13 (1), 105-110 (1977).

[6] Бештоков М.Х. К краевым задачам для вырождающихся псевдопараболических уравнений с дробной производной Герасимова-Капуто, Изв. вузов. Матем., №10, 3-16 (2018). 
[7] Самко С.Г., Килбас А.А., Маричев О.И. Интегралы и производные дробного порядка и некоторые их приложения (Минск, 1987).

[8] Самарский А.А. Теория разностных схем (Наука, М., 1983).

[9] Алиханов А.А. Априорные оченки решений краевых задач для уравнений дробного порядка, Дифференц. уравнения 46 (5), 658-664 (2010).

[10] Alikhanov A.A. A new difference scheme for the time fractional diffusion equation, J. Comput. Phys. 280, 424-438 (2015).

[11] Воеводин А.Ф., Шугрин С.М. Численные методы расчета одномерных систем (Наука, СО АН СССР, Новосибирск, 1981).

\section{Мурат Хамидбиевич Бештоков}

Институт прикладной математики и автоматизации КБНЦ РАН, 360004, г. Нальчик, ул. Шортанова, д. 89 А, Россия,

e-mail: beshtokov_murat@rambler.ru

\section{M.Kh. Beshtokov}

\section{Boundary-value problems for loaded pseudoparabolic equations of fractional order and difference methods of their solving}

Abstract. The paper is devoted to local and nonlocal boundary-value problems for loaded differential equation with variable coefficients with Gerasimov-Kaputo fractional derivative. To solve the considered problems we obtain apriori estimates in differential and difference interpretations, from which it follows the uniqueness and stability of solution with respect to initial data and right-hand side, as well as convergence of solution to difference problem to a solution to differential problems.

Keywords: boundary-value problems, apriori estimate, loaded equations, equation of pseudoparabolic type, differential equation of fractional order, Gerasimov-Kaputo fractional derivative.

Murat Khamidbievich Beshtokov

Institite of applied mathematics and automation KBSC RAS,

360004, Nalchik, Shortanova str., 89 A, Russia,

e-mail: beshtokov_murat@rambler.ru 